\title{
Coaction of Electrostatic and Hydrophobic Interactions: Dynamic Constraints on Disordered TrkA Juxtamembrane Domain
}

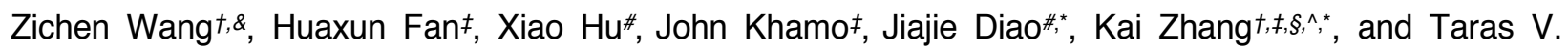
Pogorelovt,\&,\&,A,

tDepartment of Chemistry, University of Illinois at Urbana-Champaign, Urbana, Illinois 61801, USA

¥Department of Biochemistry, University of Illinois at Urbana-Champaign, Urbana, Illinois 61801, USA

\#Department of Cancer Biology, University of Cincinnati College of Medicine, Cincinnati, OH 45267, USA

$\S$ Center for Biophysics and Computational Biology, University of Illinois at Urbana-Champaign, Urbana, Illinois 61801, USA

\&National Center for Supercomputing Applications, University of Illinois at Urbana-Champaign, Urbana, Illinois 61801, USA

^Beckman Institute for Advanced Science and Technology, University of Illinois at Urbana-Champaign, Urbana, Illinois 61801, USA

\section{AUTHOR INFORMATION \\ Corresponding Authors}

*To whom correspondence may be addressed. Email: jiajie.diao@uc.edu, kaizkaiz@illinois.edu, and pogorelov@illinois.edu 


\section{ABSTRACT}

The receptor tyrosine kinase family transmits signals into cell via a single transmembrane helix and a flexible juxtamembrane domain (JMD). Membrane dynamics makes it challenging to study the structural mechanism of receptor activation experimentally. In this study, we employ all-atom molecular dynamics with Highly Mobile Membrane-Mimetic to capture membrane interactions with the JMD of tropomyosin receptor kinase $\mathrm{A}$ (TrkA). We find that $\mathrm{PIP}_{2}$ lipids engage in lasting binding to multiple basic residues and compete with salt bridge within the peptide. We discover three residues insertion into the membrane, and perturb it through computationally designed point mutations. Single-molecule experiments indicate the contribution from hydrophobic insertion is comparable to electrostatic binding, and in-cell experiments show that enhanced TrkA-JMD insertion promotes receptor ubiquitination. Our joint work points to a scenario where basic and hydrophobic residues on disordered domains interact with lipid headgroups and tails, respectively, to restrain flexibility and potentially modulate protein function.

\section{TOC GRAPHICS}
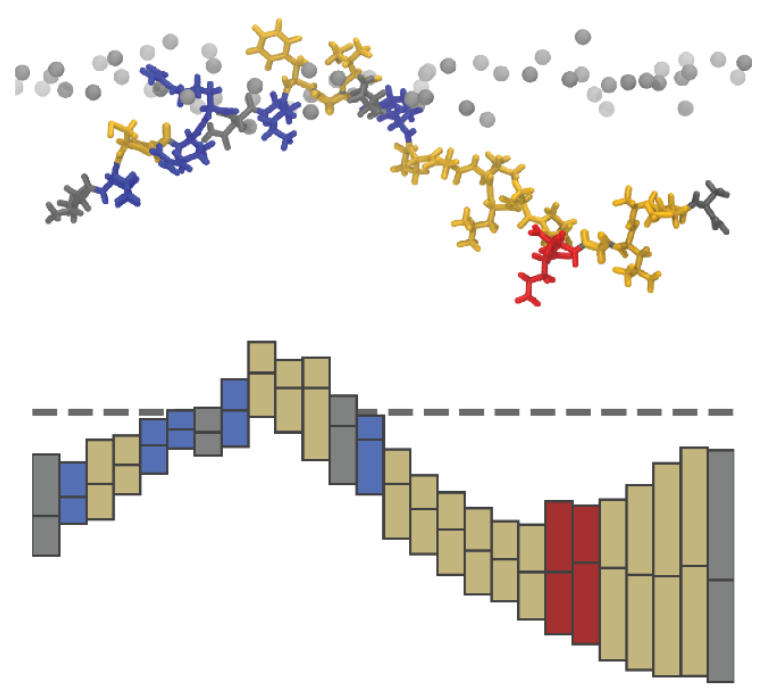

KEYWORDS signaling, receptor tyrosine kinase, lipid, molecular dynamics 


\section{Introduction}

Effective transmembrane signaling is vital to cellular decision-making. The family of receptor tyrosine kinase (RTK) contains nearly 60 membrane proteins that dimerize upon ligand binding, induce autophosphorylation in intracellular kinase domain, and activate downstream cascades that affect cell proliferation and function. RTK can be structurally divided into a extracellular kinase ligand-binding ectodomain, a single-pass transmembrane domain (TMD), a short intracellular juxtamembrane domain (JMD), and an intracellular kinase domain (Figure 1a). ${ }^{1}$ RTKs have adopted a complex regulatory role in signaling cascades ${ }^{1}$ and are subject to transactivation by other proteins, e.g. G-protein coupled receptors. ${ }^{2}$

Experimental studies have assigned unique functions for receptor activation to the $\mathrm{JMD}^{3,4}$, which can form secondary structure or remain disordered. In particular, the JMD of the epidermal growth factor receptor (EGFR) can form a membrane-anchored helix, or be part of a free antiparallel helix dimer to favor the inactive or active state, respectively. ${ }^{5,6}$ In the fibroblast growth factor receptor 3 (FGFR3), a disordered JMD is released from being embedded in the membrane upon rotation of TMD by activating mutations. ${ }^{7}$ JMD in some RTKs can also stabilize unliganded dimer ${ }^{8}$, or serve as docking site for other cellular proteins. ${ }^{9}$

Common in these studies is the interplay between JMD and cytoplasmic membrane. An extensive computational study ${ }^{10}$ of all RTK JMDs reveals that the conserved positively-charged JMD N-terminus actively interacts with anionic lipids such as phosphatidylserine (POPS) and phosphatidylinositol 4,5bisphosphate $\left(\mathrm{PIP}_{2}\right)$. The JMDs can sequester $\mathrm{PIP}_{2}$, effectively modifying local membrane environment. ${ }^{11-}$ ${ }^{13}$ Moreover, $\mathrm{PIP}_{2}$-JMD interaction has been shown to modulate receptor activity of EGFR. ${ }^{14}$
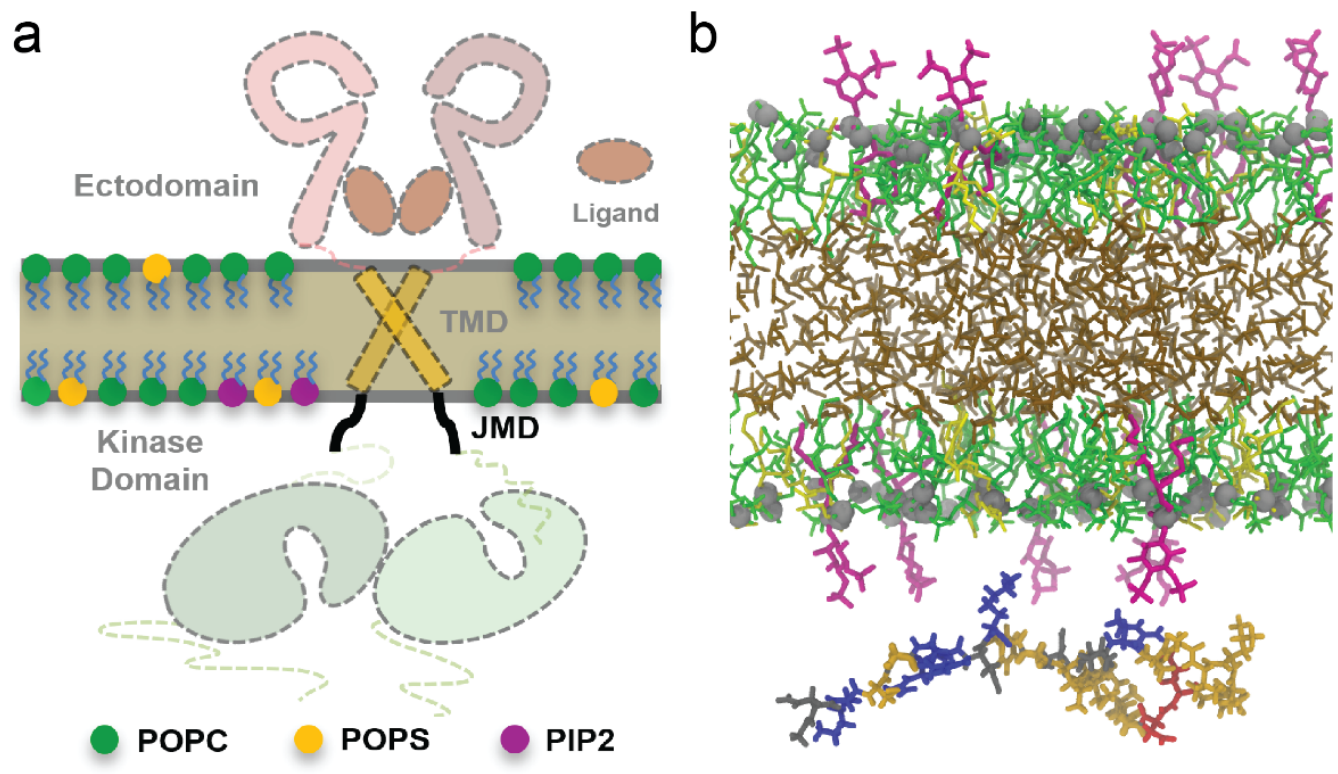

Figure 1. (a) Overview of the TrkA receptors in the active dimer form. Domains not included in modelled systems are greyed out. Structural details serve illustrative 
purpose only. (b) A snapshot of the modelled system: lipids and peptide are colorcoded by type; HMMM solvent DCLE is in brown.

Tropomyosin receptor kinase A (TrkA) is well-studied for its role in neuronal differentiation and synapse plasticity, ${ }^{15}$ but little structural information is known regarding the activation mechanism. Deletion of a conserved Lys-Phe-Gly patch in TrkA-JMD impacts signaling activity and receptor turnover, ${ }^{16}$ hinting at the significance of this flexible region.

Mechanistically, upon ligand binding a conformational change is propagated from ectodomain to TMD, which by employing JMD as a flexible fulcrum, reorients the kinase domain for autophosphorylation. It is still largely unknown how this short, intrinsically disordered JMD region transduces structural change in a controlled manner. Here we study membrane-protein interaction of TrkA-JMD using all-atom molecular dynamics (MD) with the Highly Mobile Membrane-Mimetic model (HMMM)17,18 and matching experiments. The HMMM shortens the full-length lipid tails and fills the membrane core with an organic solvent which raises lipid lateral diffusion by ten-fold, allowing enhanced conformation sampling. We used the CHARMM36m forcefield refined for intrinsically disordered peptides. ${ }^{19}$ We designed three membranes PC, $\mathrm{PC} / \mathrm{PS}$, and PC/PS/PIP 2 (Table S2) with concentrations mimicking mammalian membrane. ${ }^{20}$ Wild-type (WT) TrkA-JMD of 26 amino acids was equilibrated and placed parallel to the membrane. Five replicates are made for each membrane type and subjected to 200 ns MD simulations, totaling $3 \mu$ s modeling time. 


\section{Result}

Hydrophobic patch on JMD inserts into membranes of different compositions. To probe the conformations of TrkA-JMD bound to different membranes, we measured the distances between the membrane (mean position of glycerol phosphorus atoms) and the center of mass of each residue (Figure 2a - PC/PS/PIP ; Figure S2, S3 - PC, PC/PS). Basic residues in the N-terminus (blue) predominantly interact with lipid head groups, as evidenced by their partitioning just below the glycerol phosphorus atoms. Residues Phe448, Gly449, and lle450, however, are partitioned with the edge of lipid tails beyond the headwater interfacial region, in all three membrane types. This behavior is defined as membrane insertion in further discussion. Residues downstream of Arg452 do not interact with the membranes and are suspended in bulk water. The large interquartile range for residues on the C-terminus indicates high flexibility caused by the truncation of the kinase domain as well as inability to insert into the membrane.

\section{a}

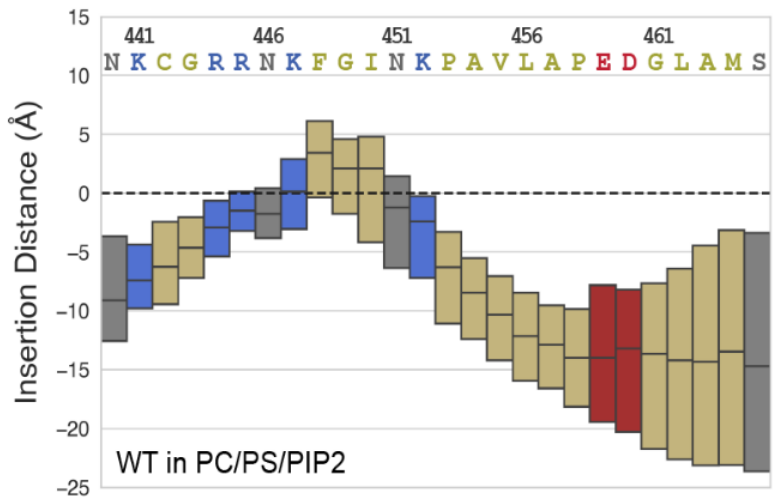

b

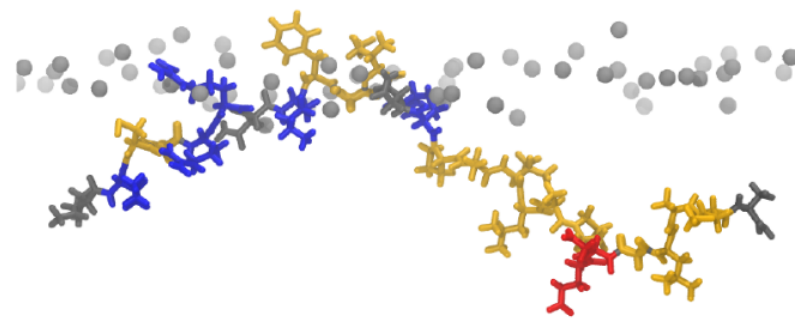

Figure 2. TrkA-JMD insertion into the membrane. (a) Distance between residue side-chain center of mass and the average position of glycerol phosphorus atoms (horizontal dashed line) in $\mathrm{PC} / \mathrm{PS} / \mathrm{PIP}_{2}$ membrane. Data points are represented in boxplot and three quartiles are shown. Boxes and the sequence are color-coded by residue type: yellow - hydrophobic, grey - polar, blue - basic, and red - acidic. (b) A snapshot of peptide insertion: membrane core is on the top; phosphorus atoms are grey spheres. 
Mutations on key residues alter JMD insertion profile. To investigate the significance of hydrophobic insertion and charge interaction, three JMD mutations were computationally designed. First, Lys447, Phe448, Gly449 were deleted (dKFG), as in experiments performed in live cells. ${ }^{16}$ Second, Phe448 was mutated to Ser448 (FS) to remove the favorable aromatic ring insertion. Third, Arg452 was substituted with Trp452 (RW) to replace charge interactions between peptide and lipid headgroups with hydrophobic interactions. Total $9 \mu$ s simulation time of the 3 mutant systems were obtained with the same protocols as the WT. In dKFG and FS, the removal of membrane-associative residues Lys447 and Phe448, or the introduction of polar residue Ser448 eliminates insertion and leaves only electrostatic anchoring. In RW, the loss of basic Arg452 is compensated by the introduced Trp452 insertion (Figure 3a). As a result, residues 448 - 456 are largely embedded within the membrane. Similar binding patterns were observed for other membrane compositions (Figures S2, S3).
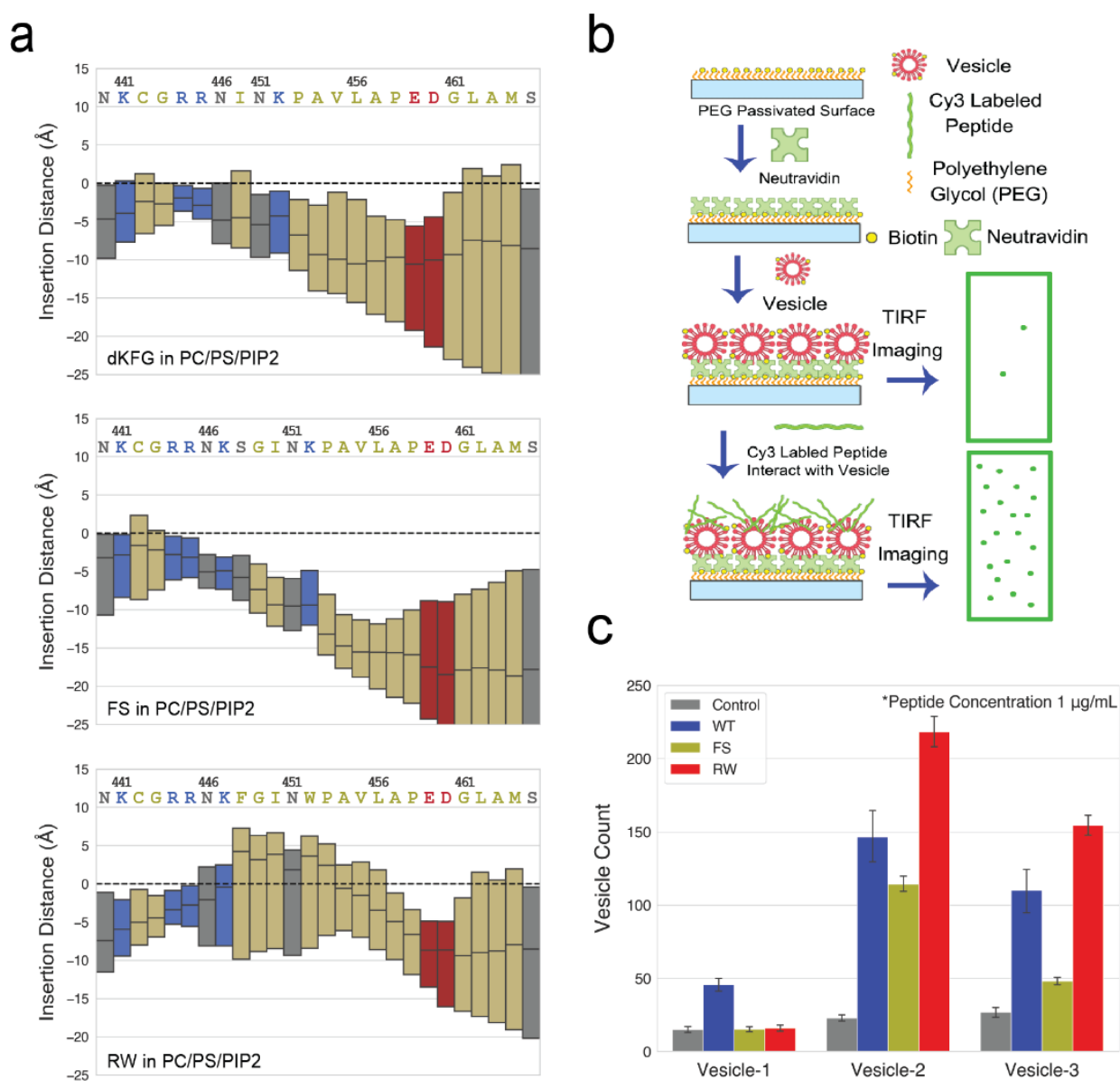

Figure 3. Role of mutations on TrkA-JMD binding. (a) Insertion distance for dKFG, $\mathrm{FS}, \mathrm{RW}$ mutants in $\mathrm{PC} / \mathrm{PS} / \mathrm{PIP}_{2}$ membrane. (b) Illustration of the surface functionalization and single-molecule imaging of specific bound peptide on vesicles immobilized on channel surface. (c) Fluorophore counts of Cy3-labeled peptides bound with different vesicles at peptide concentration equal to $1 \mu \mathrm{g} / \mathrm{mL}$. 
Peptide binding to designed vesicles supports computational results. To validate the computational findings, we used a single-molecule detection-based peptide-liposome binding assay to evaluate the binding affinity between DiD-labeled, composition-controlled lipids and Cy3-labeled JMD peptides (Figure 3b). The following three vesicle compositions were used: vesicle-1 100\% DOPC, vesicle2 65\% DOPC and $35 \%$ DOPS, and vesicle-3 95\% DOPC and 5\% PIP 2 . Full coverage vesicles were prepared to eliminate non-specific interactions between peptides and the polyethylene glycol (PEG) coated surface. The peptide-vesicle binding affinity was directly proportional to the number of Cy3 fluorescent spots in a field of view. Enhanced peptide binding was seen in PC/PS and PC/PIP 2 vesicles with $1 \mu \mathrm{g} / \mathrm{mL}$ of peptide. The number of detected spots increases from FS to WT to RW (Figure 3c), suggesting that the partition susceptibility of JMD binding to anionic membrane ranks as $\mathrm{FS}<\mathrm{WT}<\mathrm{RW}$. This result agrees with the insertion profiles predicted by MD simulations. PC-only membrane is likely too neutral for initial peptide recognition through diffusion in experiment, while the separation is small enough for electrostatic association in simulation.

Basic residues on JMD forms persistent interactions with PIP $_{2}$. To explore protein-lipid interaction as is evident from the membrane partitioning of $\mathrm{N}$-terminus, contacts between charged moieties of lipids and residues 440 - 452 were mapped. Mean contact number measures the average number of contacting lipids at a given frame. Mean contact number for the WT confirms that most contacts were formed with the basic residues (Figure 4a, see Figure S4 for mutants). Considering the amounts of lipids present are nearly equal, stronger charge interaction is formed in PC/PS, PC/PS/PIP2 membranes (on average two lipids per basic residue) than in the PC membrane (one lipid per basic residue). Mean contact duration measures the average time a lipid stays in contact with the residue side-chain before it dissociates. Mean contact duration for each type of lipid is calculated for the PC/PS/PIP2 membrane in contact with the WT (Figure 4b, see Figure S5 for mutants). The average binding time of $\mathrm{PIP}_{2}$ is on the order of tens of nanoseconds, in stark contrast with the short-lived contacts by PC and PS lipids. 
a

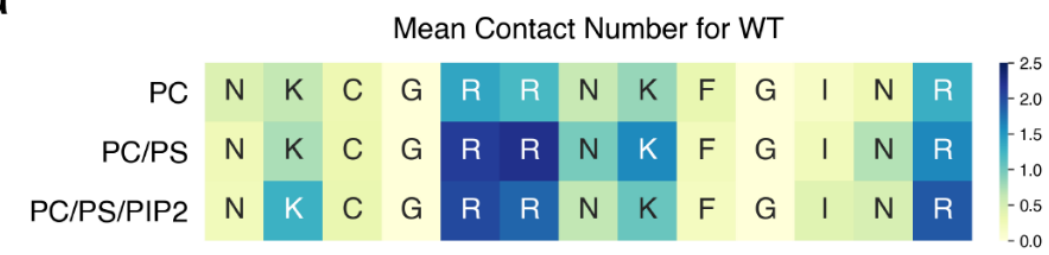

b

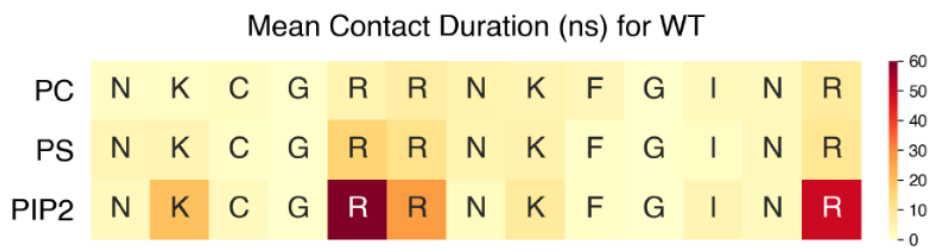
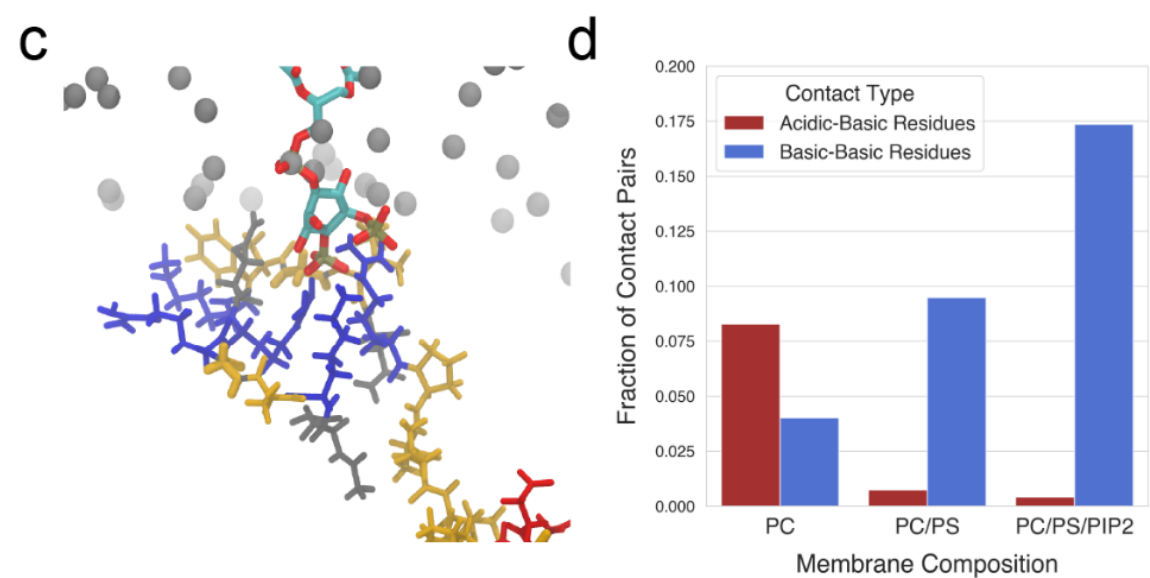

Figure 4. Molecular characterization of JMD-lipid interactions. (a) Mean contact number for 3 membranes and 13 residues in the N-terminus of WT-JMD. (b) Mean contact duration for 3 types of lipids in PC/PS/PIP 2 and 13 residues in the $\mathrm{N}$ terminus of WT-JMD. (c) A snapshot of $\mathrm{PIP}_{2}$ lipid phosphates coordinating three JMD basic residues. (d) The fraction of contact pairs for acidic-basic and basicbasic contacts on JMD (median of the 20 replicates per membrane type is shown).

Anionic lipids and acidic residues compete for binding with basic residues. To address the role of lipids with different net charges, we analyzed contacts formed between residues on JMD of the WT and mutants. The contacts were evaluated in fraction of contact pairs, the average occurrence of tworesidue contacts divided by the number of possible pairs to be formed (Figure 4d). Particularly, contacts between any of acidic Glu459, Asp460 and any of the basic residues (vary in WT and mutants) are shown to be present in PC-only membrane, but not in the more charged membranes. However, contacts between different basic residues are much more abundant in more negatively charged membranes. 
RW-TrkA mutant accelerates TrkA degradation in mammalian cells. Because TrkA-JMD is involved in NGF-mediated degradation, ${ }^{16}$ we hypothesize that mutations altering JMD-membrane interactions can affect in-cell degradation kinetics. To test this hypothesis, we transfected SH-SY5Y human neuronal cells with wild-type (WT) and mutant (FS, RW) hTrkA fused with a human influenza hemagglutinin tag (hTrkA-HA). Twenty-four hours after transfection, cells were starved in serum-free medium for 6 hours prior to treatment with nerve growth factor (NGF). Cycloheximide was applied $1 \mathrm{~h}$ before NGF treatment, and Western blot analysis against HA was used to monitor the intracellular level of TrkA. The abundance of WT- and FS-hTrkA-HA decreased to approximately 80\% 180 min after NGF treatment (Figure 5a). The RW mutant, on the other hand, showed significantly faster degradation kinetics, as evidenced by the $50 \%$ pre-treatment level as short as 90 min after NGF treatment (Figure 5b). Variation of the hTrkA abundance should not arise from unequal loading because the level of the GAPDH (loading control) protein from the same blot were comparable between replicates. This result suggested that RW mutation accelerates the NGF-mediated degradation kinetics of hTrkA, given that an equal amount of WT and mutant hTrkA was transfected.
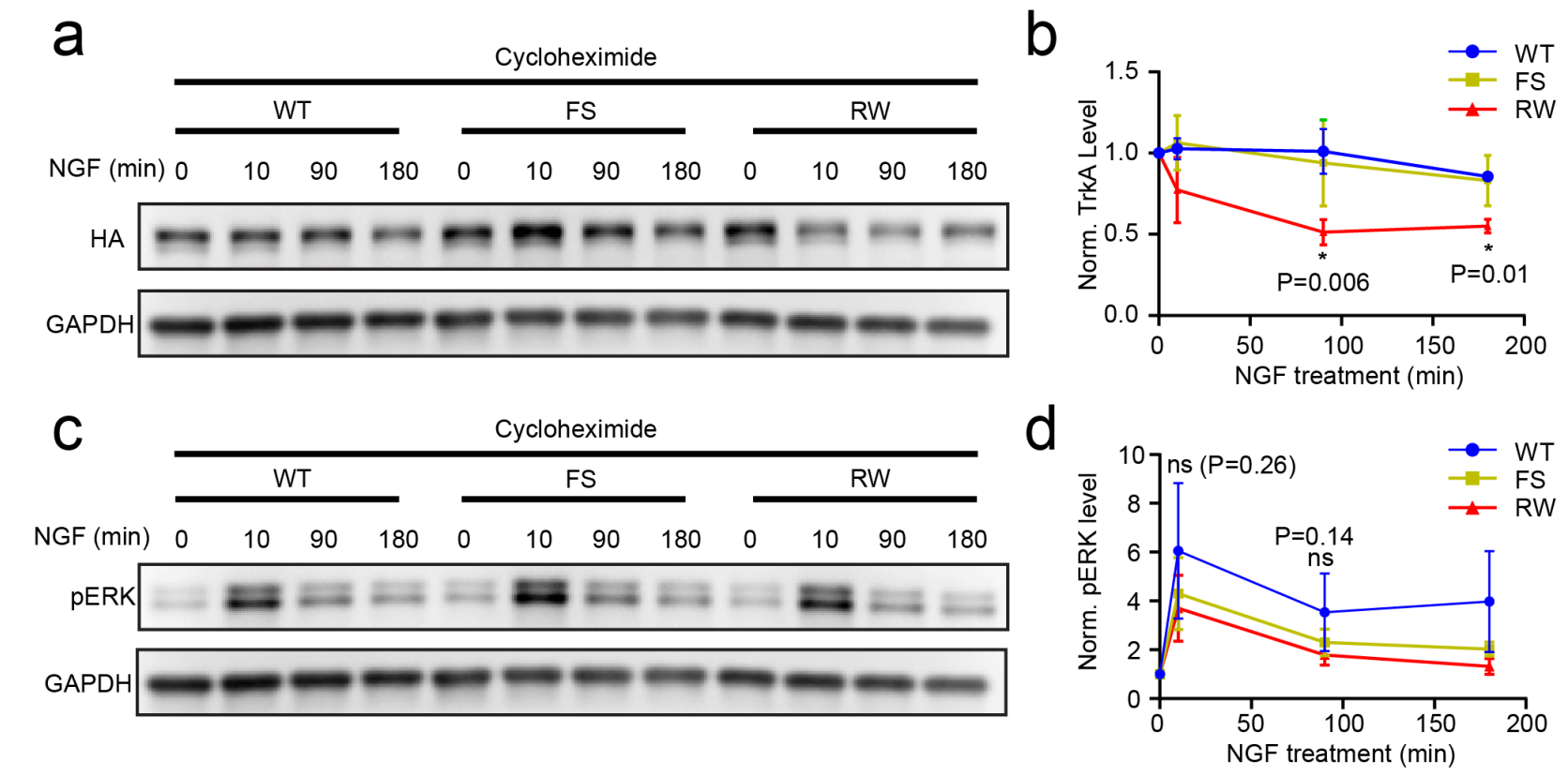

Figure 5. Quantitative determination of the degradation kinetics and signaling activity for the WT, FS, and RW HAhTrkA mutants in SHSY5Y cells. (a) Western blot analysis of TrkA abundance probed with an anti-HA antibody. Serumdeprived cells were pre-treated with cycloheximide to block new protein synthesis, followed by $100 \mathrm{ng} / \mathrm{mL} \mathrm{NGF}$ treatment for 0, 10, 90, and $180 \mathrm{~min}$. GAPDH was used as a loading control. (b) Quantification of the TrkA level normalized over GAPDH level from three replicates. The RW mutant shows significantly faster degradation kinetics than the WT and FS mutants. (c) Western blot analysis of the NGF-mediated ERK signaling activation in SHSY5Y cells. 
Cells were treated the same as in a). (d) Quantification of the pERK level normalized over GAPDH level from three replicates. No significant difference was detected between WT and two TrkA mutants.

TrkA mutants maintain the same short-term signaling capacity as the wild-type. NGF-TrkA binding leads to activation of the downstream Ras/ERK (extracellular signal-regulated kinase) signaling pathway within $10 \mathrm{~min}$. To determine if mutants hTrkA are functional, we measured the fold increase of pERK level upon NGF stimulation. FS and RW mutants show similar fold increase of pERK level as WTTrkA (Figure 5c-d). To account for background noise from indigenous TrkA in SH-SY5Y cell-line, we repeat in HEK293 cell-line that does not express indigenous TrkA or degradation machinery. The results confirm that the difference in the signaling capacity of WT/FS/RW-TrkA cannot be resolved from noise (Figure S9). 


\section{Discussion}

TrkA-JMD conformation is distinct from experimentally ${ }^{3}$ and computationally ${ }^{6}$ characterized EGFRJMD conformations: EGFR-JMD forms a short but structured 9-residue helix from Leu679 to Glu687 that can be membrane-embedded, while the corresponding lle450 to Pro458 residues in TrkA-JMD are disordered and suspended in solvent. In the $\mathrm{N}$-terminus, in addition to strong electrostatic binding enabled by the common presence of basic residues, TrkA-JMD possesses stable hydrophobic insertion unseen in EGFR-JMD. Free energy changes of side-chains insertion into HMMM agrees well with values into full-tail membrane. ${ }^{21}$ The insertions characterized by MD-HMMM simulations can also be validated in light of the experimental Wimley-White hydrophobicity scale. ${ }^{22}$ The Wimley-White scale provides the free energy change of partitioning the whole residue into POPC membrane using pentapeptide AcWL-X-LL, describing the tendency of $X$ for membrane insertion. Basic residue associating with anionic membrane is a different scenario and is not expected to follow the positive free energies given by the scale.

For some relevant residues, Phe and Trp, have negative free energies of -1.13 and $-1.85 \mathrm{kcal} / \mathrm{mol}$, respectively. Ser, Pro, Ala, Val have positive free energies of $0.13,0.45,0.17,0.07 \mathrm{kcal} / \mathrm{mol}$, respectively, due to the unfavorable contribution from polar peptide bonds. These free energy values are consistent with the following computational results: 1) in WT, Phe448 engages in stable insertion, yet residues after Arg452 do not; 2) FS mutation completely removes insertion; 3) RW mutation is highly favorable and compensate for the loss of basic-lipid interaction and even promotes insertion of additional residues. Though the WimleyWhite scale is obtained for POPC membrane, similar patterns of insertion exist in more anionic membranes. Altogether, our results suggest that hydrophobic insertion also contributes to the membrane anchoring and stabilization of $\mathrm{N}$-terminus TrkA-JMD, comparable to previously characterized electrostatic binding ${ }^{7,10-13}$ between JMD and lipids.

In all three lipid compositions, JMD peptide rapidly forms an encounter complex with membrane from the initial position $20 \AA$ away. This membrane attachment is likely the effect of electrostatics between anionic lipids and the basic residues in JMD. This long-range charge-interaction is necessary to anchor the JMD N-terminus, and allow membrane insertion. Although PS and $\mathrm{PIP}_{2}$ lipids exhibit enhanced charge interaction (Figure 4a), PS lipids tend to form frequent yet transient contacts, while $\mathrm{PIP}_{2}$ lipids persistently bind to basic residues (Figure $4 \mathrm{~b}$ ). With the addition of anionic lipids, fold-increase of basic-basic residue contacts in JMD is observed (Figure 4d). Combined with the contact analysis, the results point to a distinct mode of $\mathrm{PIP}_{2}$-peptide interactions where the two phosphate groups on the sugar ring coordinate more than one basic residue for prolonged time (tens of nanoseconds).

The major effect of membrane composition on peptide conformation can be seen on JMD Cterminus, marked by the presence of acidic residues. This region tends to be more flexible and distal from the membrane with increased amounts of anionic lipids (Figure 2a, 3a, S2, S3). One factor is the repulsion 
between acidic residues and enriched negative charge in the membrane. Another factor is the competition between anionic lipids and JMD acidic residues to bind basic residues. As more anionic lipids are included, contacts between acidic and basic residues that loop the C-terminus closer to membrane gets depleted (Figure 4d). This effect would be significant if the orientation of JMD C-terminus in turn affects orientation of the downstream kinase domain. The interplay between membrane composition and JMD orientation might potentially explain the relative conservation of acidic residues on the C-terminus region of JMD in all RTKs. ${ }^{10}$

Endocytosis of TrkA that results in endosome degradation or receptor recycling is triggered by NGF-induced ubiquitination performed by multiple E3 ligases.23-25 Lys447 is mono-ubiquitinated and its deletion causes resistance to degradation. ${ }^{16,25}$ Our insertion analysis shows that Lys447 is membrane inserted for half of the simulation time in the WT and RW (likely driven by Phe448), and is mostly free in the FS. Intriguingly, our in-cell results suggest the level of ubiquitination on FS-TrkA is similar to WT-TrkA (Figure 5b), even if Lys447 is more exposed to solvent. The finding that RW mutation promotes ubiquitination is subject to two possibilities: 1) the insertion profile of residues downstream Lys447 affects substrate recognition of the E3 ligase, likely Cbl-b;25 2) as ubiquitination occurs after receptor activation, RW mutation changes the activated JMD conformation to be more prone to ubiquitination. This finding can guide future studies that probe the details of ubiquitination by E3 ligase, and TrkA conformational changes upon activation.

Prior studies suggest that JMDs in EGFR and FGFR3 can switch from membrane-embedded state to free-floating state, depending on TMD dimer tilt angles. ${ }^{5-7}$ Inspired by these findings, we hypothesize that mutant FS or RW changes inherent TrkA activity by reducing or enhancing JMD binding affinity to the membrane, shifting equilibrium to favor one state. Previously published experiments showed that the dKFG mutation induces more robust signaling, ${ }^{16}$ which correlates with our hypothesis and computational observation of reduced membrane binding (Figure 3a). However, our in-cell experiments show that the difference in the signaling capacity of WT/FS/RW-TrkA is not significantly different. Although here we computationally reported the behavior of isolated TrkA-JMD, our experiments suggest the role of JMD in full receptor can be more involved and requires further investigation. 


\section{Conclusion}

Using all-atom molecular dynamics, we highlight the roles of hydrophobic and electrostatic binding in peptide-membrane interactions. We characterized charge-driven interaction between anionic lipids and TrkA-JMD N-terminus basic residues. We found $\mathrm{PIP}_{2}$ engages in persistent binding to multiple basic residues and can compete with electrostatic binding within the peptide. In addition, we revealed a significant contribution from hydrophobic insertion in membrane-anchoring. We were able to either diminish or increase the level of insertion by mutations. Peptide-binding experiments support computational results, while biochemical assays indicate TrkA-JMD has a more convoluted role in signaling. Our findings also provide insights into receptor turnover through ubiquitination. 


\section{ASSOCIATED CONTENT}

Supporting Information. Supporting information contains supplementary methods, supplementary figures, and supplementary tables.

\section{AUTHOR INFORMATION}

Notes

The authors declare no competing financial interests.

\section{ACKNOWLEDGMENT}

T. V. P. acknowledges support from the Department of Chemistry, the School of Chemical Sciences, and the Office of the Vice-Chancellor for Research (RSOCR Award 4703) at University of Illinois at UrbanaChampaign. T. V. P. acknowledges computational resources from the Texas Advanced Computing Center (TACC) at the University of Texas at Austin made available from the Extreme Science and Engineering Discovery Environment (XSEDE) Grant TG-MCB130112, which is supported by National Science Foundation Grant ACl-1053575. K. Z. acknowledges support from the School of Molecular and Cellular Biology at UIUC and NIH (1R01GM132438). J.D. acknowledges support from NIH (R35GM128837). Z. W. acknowledges support from Student Pushing Innovation (SPIN) Program of the National Center for Supercomputing Applications (NCSA). Authors are grateful to Dr. Yekaterina (Katka) Golubeva for assistance with the TrkA schematics. 


\section{REFERENCES}

(1) Lemmon, M. A.; Schlessinger, J. Cell Signaling by Receptor-Tyrosine Kinases. Cell 2010, 141 (7), 1117-1134. https://doi.org/10.1016/j.cell.2010.06.011.

(2) Zhou, R.; Han, B.; Xia, C.; Zhuang, X. Membrane-Associated Periodic Skeleton Is a Signaling Platform for RTK Transactivation in Neurons. Science 2019, 365 (6456), 929-934. https://doi.org/10.1126/science.aaw5937.

(3) Jura, N.; Endres, N. F.; Engel, K.; Deindl, S.; Das, R.; Lamers, M. H.; Wemmer, D. E.; Zhang, X.; Kuriyan, J. Mechanism for Activation of the EGF Receptor Catalytic Domain by the Juxtamembrane Segment. Cell 2009, 137 (7), 1293-1307. https://doi.org/10.1016/j.cell.2009.04.025.

(4) He, L.; Hristova, K. Consequences of Replacing EGFR Juxtamembrane Domain with an Unstructured Sequence. Sci. Rep. 2012, 2, 854. https://doi.org/10.1038/srep00854.

(5) Endres, N. F.; Das, R.; Smith, A. W.; Arkhipov, A.; Kovacs, E.; Huang, Y.; Pelton, J. G.; Shan, Y.; Shaw, D. E.; Wemmer, D. E.; et al. Conformational Coupling across the Plasma Membrane in Activation of the EGF Receptor. Cell 2013, 152 (3), 543-556.

https://doi.org/10.1016/j.cell.2012.12.032.

(6) Arkhipov, A.; Shan, Y.; Das, R.; Endres, N. F.; Eastwood, M. P.; Wemmer, D. E.; Kuriyan, J.; Shaw, D. E. Architecture and Membrane Interactions of the EGF Receptor. Cell 2013, 152 (3), 557-569. https://doi.org/10.1016/j.cell.2012.12.030.

(7) Tamagaki, H.; Furukawa, Y.; Yamaguchi, R.; Hojo, H.; Aimoto, S.; Smith, S. O.; Sato, T. Coupling of Transmembrane Helix Orientation to Membrane Release of the Juxtamembrane Region in FGFR3. Biochemistry 2014, 53 (30), 5000-5007. https://doi.org/10.1021/bi500327q.

(8) Sarabipour, S.; Hristova, K. FGFR3 Unliganded Dimer Stabilization by the Juxtamembrane Domain. J. Mol. Biol. 2014, 427 (8), 1705-1714. https://doi.org/10.1016/j.jmb.2015.02.013.

(9) Sengupta, P.; Bosis, E.; Nachliel, E.; Gutman, M.; Smith, S. O.; Mihályné, G.; Zaitseva, I.; McLaughlin, S. EGFR Juxtamembrane Domain, Membranes, and Calmodulin: Kinetics of Their Interaction. Biophys. J. 2009, 96 (12), 4887-4895. https://doi.org/10.1016/j.bpj.2009.03.027.

(10) Hedger, G.; Sansom, M. S. P.; Kolds $\varnothing, ~ H$. The Juxtamembrane Regions of Human Receptor Tyrosine Kinases Exhibit Conserved Interaction Sites with Anionic Lipids. Sci. Rep. 2015, 5, 9198. https://doi.org/10.1038/srep09198.

(11) McLaughlin, S.; Smith, S. O.; Hayman, M. J.; Murray, D. An Electrostatic Engine Model for 
Autoinhibition and Activation of the Epidermal Growth Factor Receptor (EGFR/ErbB) Family. J. Gen. Physiol. 2005, 126 (1), 41-53. https://doi.org/10.1085/jgp.200509274.

(12) Halim, K. B. A.; Koldsø, H.; Sansom, M. S. P. Interactions of the EGFR Juxtamembrane Domain with PIP2-Containing Lipid Bilayers: Insights from Multiscale Molecular Dynamics Simulations. Biochim. Biophys. Acta 2015, 1850 (5), 1017-1025. https://doi.org/10.1016/j.bbagen.2014.09.006.

(13) Chavent, M.; Karia, D.; Kalli, A. C.; Domański, J.; Duncan, A. L.; Hedger, G.; Stansfeld, P. J.; Seiradake, E.; Jones, E. Y.; Sansom, M. S. P. Interactions of the EphA2 Kinase Domain with PIPs in Membranes: Implications for Receptor Function. Structure 2018, 26 (7), 1025-1034. https://doi.org/10.1016/j.str.2018.05.003.

(14) Michailidis, I. E.; Rusinova, R.; Georgakopoulos, A.; Chen, Y.; lyengar, R.; Robakis, N. K.; Logothetis, D. E.; Baki, L. Phosphatidylinositol-4,5-Bisphosphate Regulates Epidermal Growth Factor Receptor Activation. Pflugers Arch. Eur. J. Physiol. 2011, 461 (3), 387-397. https://doi.org/10.1007/s00424-010-0904-3.

(15) Huang, E. J.; Reichardt, L. F. Trk Receptors: Roles in Neuronal Signal Transduction. Annu. Rev. Biochem. 2003, 72 (1), 609-642. https://doi.org/10.1146/annurev.biochem.72.121801.161629.

(16) Kiris, E.; Wang, T.; Yanpallewar, S.; Dorsey, S. G.; Becker, J.; Bavari, S.; Palko, M. E.; Coppola, V.; Tessarollo, L. TrkA in Vivo Function Is Negatively Regulated by Ubiquitination. J. Neurosci. 2014, 34 (11), 4090-4098. https://doi.org/10.1523/JNEUROSCI.4294-13.2014.

(17) Ohkubo, Y. Z.; Pogorelov, T. V.; Arcario, M. J.; Christensen, G. A.; Tajkhorshid, E. Accelerating Membrane Insertion of Peripheral Proteins with a Novel Membrane Mimetic Model. Biophys. J. 2012, 102 (9), 2130-2139. https://doi.org/10.1016/j.bpj.2012.03.015.

(18) Vermaas, J. V.; Baylon, J. L.; Arcario, M. J.; Muller, M. P.; Wu, Z.; Pogorelov, T. V.; Tajkhorshid, E. Efficient Exploration of Membrane-Associated Phenomena at Atomic Resolution. J. Membr. Biol. 2015, 248 (3), 563-582. https://doi.org/10.1007/s00232-015-9806-9.

(19) Huang, J.; Rauscher, S.; Nawrocki, G.; Ran, T.; Feig, M.; De Groot, B. L.; Grubmüller, H.; MacKerell, A. D. CHARMM36m: An Improved Force Field for Folded and Intrinsically Disordered Proteins. Nat. Methods 2016, 14, 71-73. https://doi.org/10.1038/nmeth.4067.

(20) Van Meer, G.; Voelker, D. R.; Feigenson, G. W. Membrane Lipids: Where They Are and How They Behave. Nat. Rev. Mol. Cell Biol. 2008, 9 (2), 112-114. https://doi.org/10.1038/nrm2330.

(21) Pogorelov, T. V.; Vermaas, J. V.; Arcario, M. J.; Tajkhorshid, E. Partitioning of Amino Acids into a Model Membrane: Capturing the Interface. J. Phys. Chem. B 2014, 118 (6), 1481-1492. 
https://doi.org/10.1021/jp4089113.

(22) Wimley, W. C.; White, S. H. Experimentally Determined Hydrophobicity Scale for Proteins at Membrane Interfaces. Nat. Struct. Biol. 1996, 3 (10), 842-848. https://doi.org/10.1038/nsb1096842.

(23) Arévalo, J. C.; Waite, J.; Rajagopal, R.; Beyna, M.; Chen, Z. Y.; Lee, F. S.; Chao, M. V. Cell Survival through Trk Neurotrophin Receptors Is Differentially Regulated by Ubiquitination. Neuron 2006, 50 (4), 549-559. https://doi.org/10.1016/j.neuron.2006.03.044.

(24) Takahashi, Y.; Shimokawa, N.; Esmaeili-Mahani, S.; Morita, A.; Masuda, H.; Iwasaki, T.; Tamura, J.; Haglund, K.; Koibuchi, N. Ligand-Induced Downregulation of TrkA Is Partly Regulated through Ubiquitination by Cbl. FEBS Lett. 2011, 585 (12), 1741-1747. https://doi.org/10.1016/j.febslet.2011.04.056.

(25) Emdal, K. B.; Pedersen, A. K.; Bekker-Jensen, D. B.; Tsafou, K. P.; Horn, H.; Lindner, S.; Schulte, J. H.; Eggert, A.; Jensen, L. J.; Francavilla, C.; et al. Temporal Proteomics of NGF-TrkA Signaling Identifies an Inhibitory Role for the E3 Ligase Cbl-b in Neuroblastoma Cell Differentiation. Sci. Signal. 2015, 8 (374), ra40. https://doi.org/10.1126/scisignal.2005769. 
Supplementary Information

\section{Coaction of Electrostatic and Hydrophobic Interactions: Dynamic Constraints on Disordered TrkA Juxtamembrane Domain}

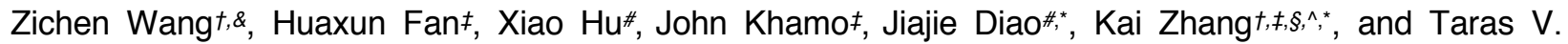
Pogorelovt,\&,\&,^,*

tDepartment of Chemistry, University of Illinois at Urbana-Champaign, Urbana, Illinois 61801, USA

¥Department of Biochemistry, University of Illinois at Urbana-Champaign, Urbana, Illinois 61801, USA

\#Department of Cancer Biology, University of Cincinnati College of Medicine, Cincinnati, OH 45267, USA

$\S$ Center for Biophysics and Computational Biology, University of Illinois at Urbana-Champaign, Urbana, Illinois 61801, USA

\&National Center for Supercomputing Applications, University of Illinois at Urbana-Champaign, Urbana, Illinois 61801, USA

'Beckman Institute for Advanced Science and Technology, University of Illinois at Urbana-Champaign, Urbana, Illinois 61801, USA

\section{AUTHOR INFORMATION}

\section{Corresponding Authors}

*To whom correspondence may be addressed. Email: jiajie.diao@uc.edu, kaizkaiz@illinois.edu, and pogorelov@illinois.edu 


\section{S-1 All-Atom MD Simulations}

\section{System Assembly}

Wild-type sequence of human-TrkA was obtained from the UniProt Knowledgebase. ${ }^{1}$ The JMD sequence was taken as the 26 amino acids C-terminal of hydrophobic transmembrane domain, and included residues 440-465. The coordinate files for the wild type and three mutants were assembled using Avogadro software, ${ }^{2}$ and then solvated in VMD and simulated for $5 \mathrm{~ns}$ for equilibration.

Table S1. TrkA-JMD Sequences

\begin{tabular}{|c|c|}
\hline & 456 \\
\hline WT & N-K-C-G-R-R-N-K-F-G-I-N-R-P-A-V-L-A-P-E-D-G-L-A-M-S \\
\hline dKFG & N-K-C-G-R-R-N ------- I-N-R-P-A-V-L-A-P-E-D-G-L-A-M-S \\
\hline FS & N-K-C-G-R-R-N-K-S-G-I-N-R-P-A-V-L-A-P-E-D-G-L-A-M-S \\
\hline RW & N-K-C-G-R-R-N-K-F-G-I-N-W-P-A-V-L-A-P-E-D-G-L-A-M-S \\
\hline
\end{tabular}

Complete peptide-membrane systems were generated using webserver CHARMM-GUI. ${ }^{3}$ Chargeneutral ACE and CT2 terminal patches were applied to the equilibrated peptide. Peptide was placed parallel and $20 \AA$ away from the membrane (Figure S1). Membranes were modeled by HMMM,4-6 and were built with HMMM Membrane Builder. ${ }^{7}$ Total 3 lipid compositions were employed. Number of POPC:POPS:PIP 2 were 59:0:0, 40:20:0, 41:12:6 per leaflet for the three compositions, respectively. Membrane $x-/ y-$ dimensions of resulting systems were fixed at $65 \AA$ × $65 \AA$, and z-dimension at $120 \AA$. Extended z-dimension serves to shield charges from the other periodic membrane leaflet. Neutralizing potassium or chloride ions were added. Each replicate was $\sim 45,000$ atoms. For statistics, 5 replicates were made for each lipid composition and JMD peptide variant (WT, dKFG, FS, RW), leading to the total of 60 replicates (Table S3). Each replicate was individually generated from CHARMM-GUI with randomization in initial configuration of lipids for better sampling. 


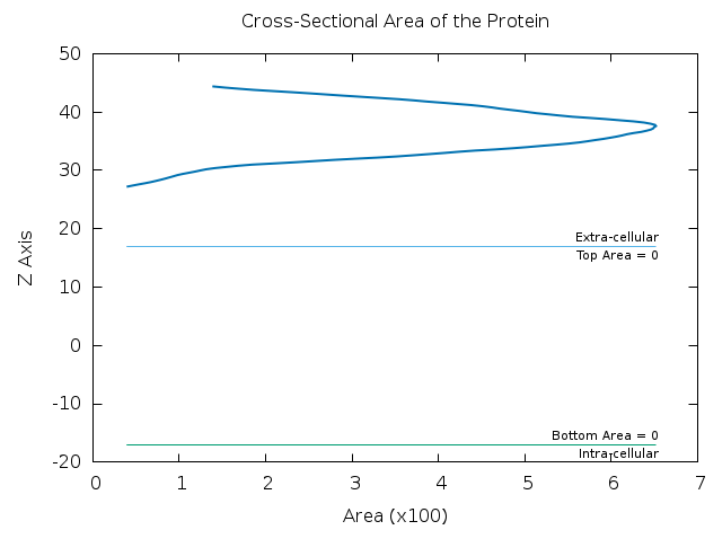

Figure S1. Initial position of peptide relative to membrane. Highest density was seen at around $z=37 \AA$ while membrane-water interface was at $z=17 \AA$.

\section{Molecular Dynamics Simulations}

All simulations were performed using the molecular dynamics software NAMD2. ${ }^{8}$ Lipids, ions, and peptides were modeled with $\mathrm{CHARMM} 32 \mathrm{~m}$ force field optimized for disordered proteins, ${ }^{9}$ water molecules with the TIP3P model, ${ }^{10}$ and DCLE molecules with CGenFF. ${ }^{11} \mathrm{~A}$ restraint of $1 \mathrm{kcal} \mathrm{mol}^{-1} \AA^{-2}$ perpendicular to membrane plane was applied to the carboxyl carbon atoms of each lipid tail to avoid short-tail HMMM lipids partitioning into the solution. The restraint allows for $\pm 3.5 \AA$ vertical motion along the $z$-directions. The Lateral motions of lipid was free of restraints. Short-range electrostatics and Van der Waals interactions were set with cutoff $12 \AA$ with switching at $10 \AA$. Long-range electrostatics was modelled by the Particle Mesh Ewald method with $1 \AA$ grid spacing. ${ }^{12}$ SETTLE algorithm was used to restrain the hydrogen-atom bond length. ${ }^{13}$ NPT ensemble was chosen. Temperature was controlled at $303.15 \mathrm{~K}$ and pressure was controlled at $1 \mathrm{~atm}$ by Langevin dynamics. ${ }^{14}$ The integration step was set at $2 \mathrm{fs}$. All equilibration MD simulations before the production followed the 6 cycles suggested by CHARMM-GUI where force constants were gradually reduced. 
Table S2. MD simulation membrane composition

\begin{tabular}{|l|l|l|l|l|}
\hline $\begin{array}{l}\text { Membrane } \\
\text { Name }\end{array}$ & $\begin{array}{l}\text { \#POPC } \\
\text { per } \\
\text { leaflet }\end{array}$ & $\begin{array}{l}\text { \#POPS } \\
\text { per } \\
\text { leaflet }\end{array}$ & $\begin{array}{l}\text { \#PIP } 2 \\
\text { per } \\
\text { leaflet }\end{array}$ & $\begin{array}{l}\text { \#lipids } \\
\text { per } \\
\text { leaflet }\end{array}$ \\
\hline PC & 57 & 0 & 0 & 57 \\
\hline PC/PS & 40 & 20 & 0 & 60 \\
\hline PC/PS/PIP2 & 41 & 12 & 6 & 59 \\
\hline
\end{tabular}

Table S3. MD simulation data summary

\begin{tabular}{|l|l|l|l|l|l|}
\hline System Name & $\begin{array}{l}\text { \#replicates } \\
\text { with } \\
\text { PC }\end{array}$ & $\begin{array}{l}\text { \#replicates } \\
\text { with } \\
\text { PC/PS }\end{array}$ & $\begin{array}{l}\text { \#replicates } \\
\text { with } \\
\text { PC/PS/PIP }\end{array}$ & $\begin{array}{l}\text { run time } \\
\text { (ns) }\end{array}$ & $\begin{array}{l}\text { total } \\
\text { \#replicates }\end{array}$ \\
\hline WT-TrkA-JMD & 5 & 5 & 5 & 200 & 15 \\
\hline dKFG-TrkA-JMD & 5 & 5 & 5 & 200 & 15 \\
\hline FS-TrkA-JMD & 5 & 5 & 5 & 200 & 15 \\
\hline RW-TrkA-JMd & 5 & 5 & 5 & 200 & 15 \\
\hline
\end{tabular}




\section{S-2 Simulation Analysis}

All trajectories were visually inspected in VMD. ${ }^{15}$ Figures $1 b, 2 b, 4 c$ were rendered in VMD. Quantitative analyses were written in Python package MDAnalysis, ${ }^{16}$ and plotted with Python package Seaborn.

1) Insertion Distance: Insertion distance is the difference between z-axis (perpendicular to membrane) position of residue center of mass and average position of all lipid glycerol phosphorus atoms on the leaflet closer to peptide, such that positive values indicate membrane insertion. Data points were taken in the last $50 \mathrm{~ns}$ with a step size of $1 \mathrm{~ns}$ from all replicates.

2) Mean Contact Number: Contact is defined as any of the lipid negatively charged groups is within $5 \AA$ of the residue side-chain. Mean contact number measures the average number of lipids in contact with the residue at one frame. The values are calculated from the last $100 \mathrm{~ns}$ with a step size of $1 \mathrm{~ns}$, and averaged in all replicates.

3) Mean Contact Duration: Contact is defined as any of the lipid negatively charged groups is within $5 \AA$ of the residue side-chain. Association period is the consecutive frames that a lipid is in contact with the residue until the contact breaks. Mean contact duration is the average time of association period (time interval size times number of frames) for all lipid-residue contacts. The values are calculated from the last $100 \mathrm{~ns}$ with a step size of $1 \mathrm{~ns}$, and averaged in all replicates.

4) Fraction of Contact Pairs: Contact is defined as the distance between the terminal integercharge groups of two residues is less than $5 \AA$. Fraction of contact pairs is the average number of detected contacts divided by the number of possible pairs. For acidic and basic residues in WT-TrkA-JMD, contacts can be formed between two acidic residues and five basic residues, total ten possible pairs. For all basic residues in WT-TrkA-JMD, contacts can be formed between any two of the five basic, total ten possible pairs. The values are calculated in the last $100 \mathrm{~ns}$ with a step size of $1 \mathrm{~ns}$ for all replicates. Median of all 20 replicates per membrane is reported. 


\section{S-3 Simulation Analysis Supplementary Figures}

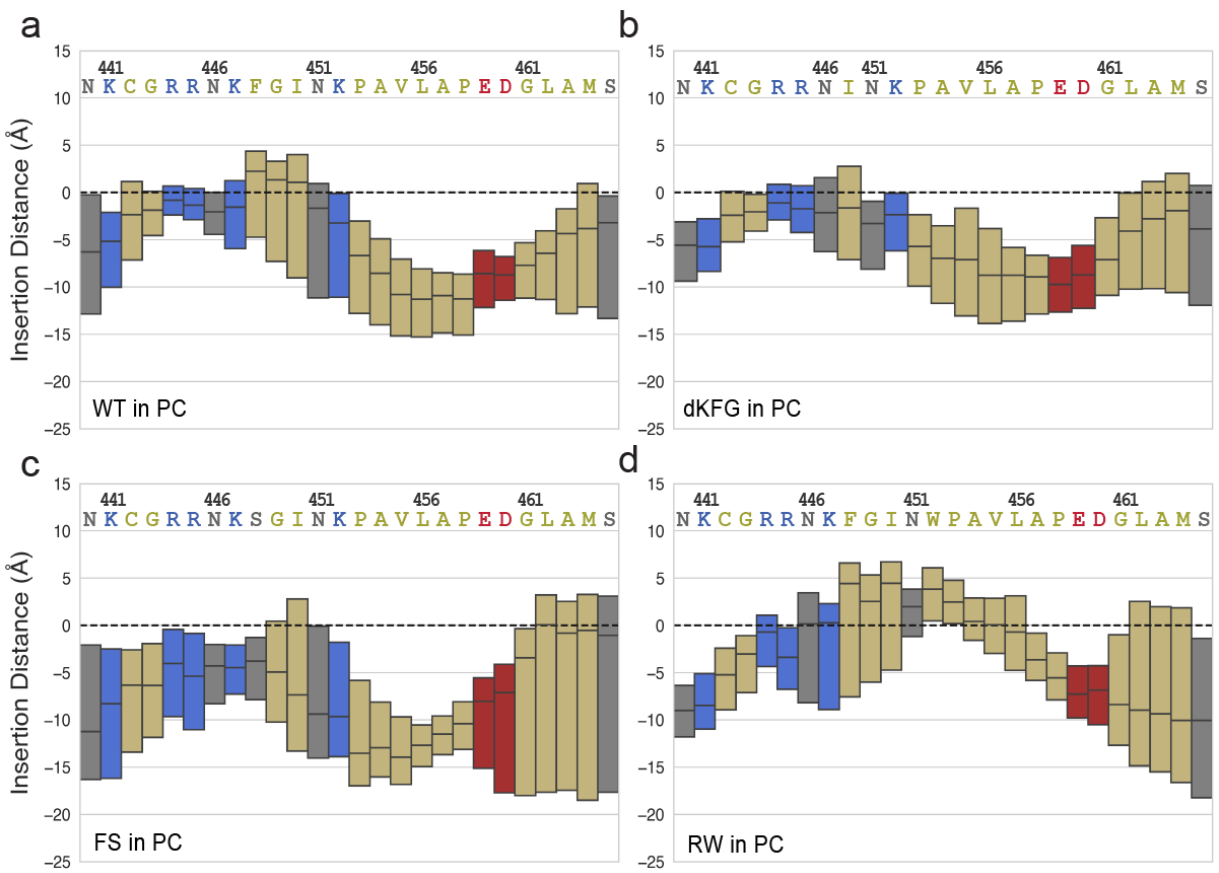

Figure S2. Insertion distance for a) WT, b) dKFG, c) FS, d) RW in PC

membrane.

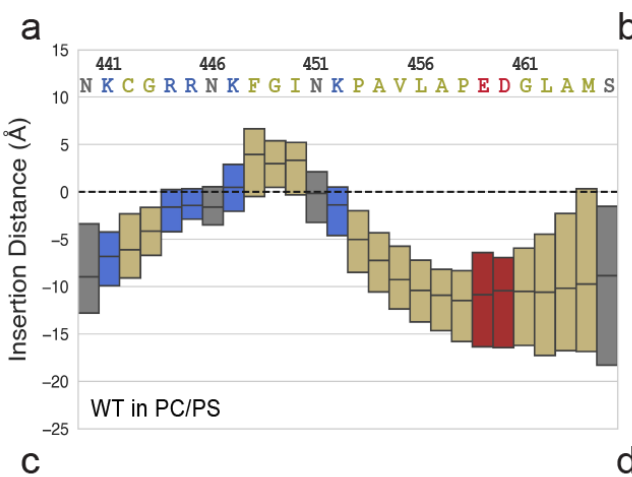

b
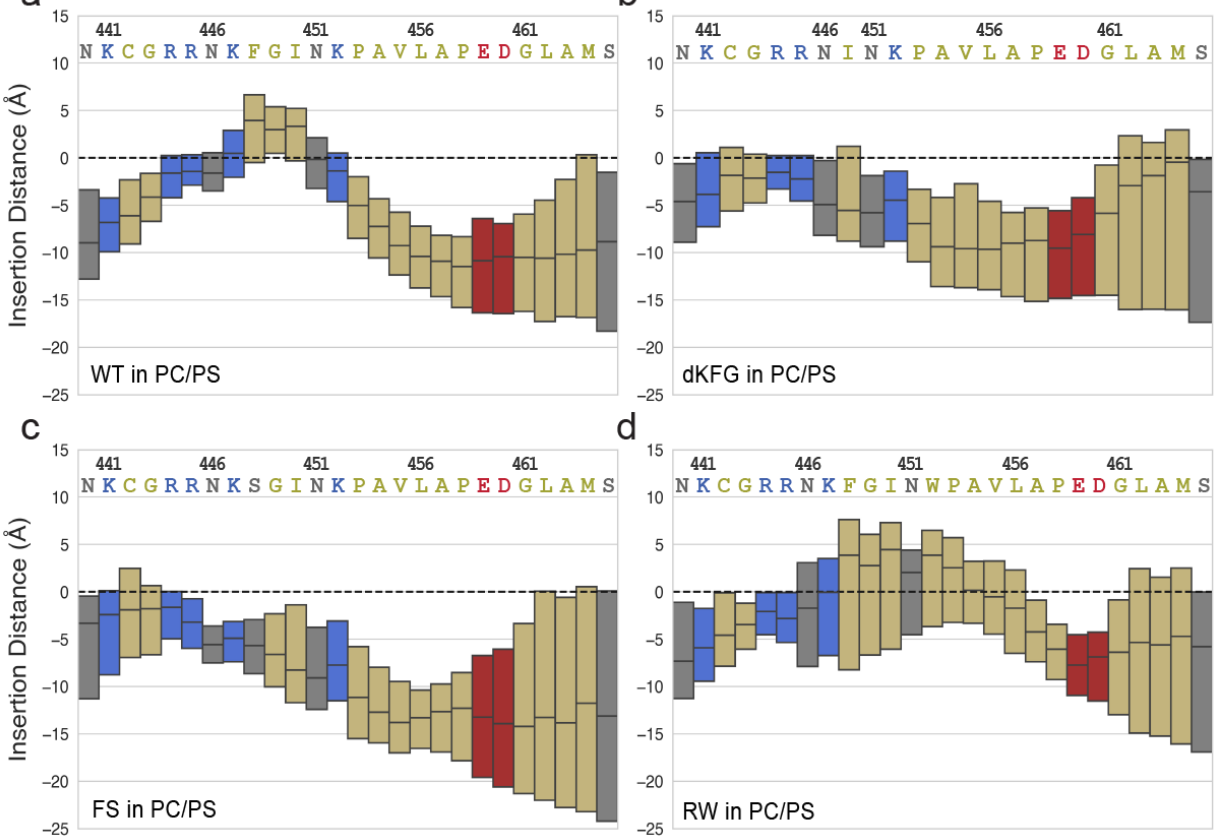

d

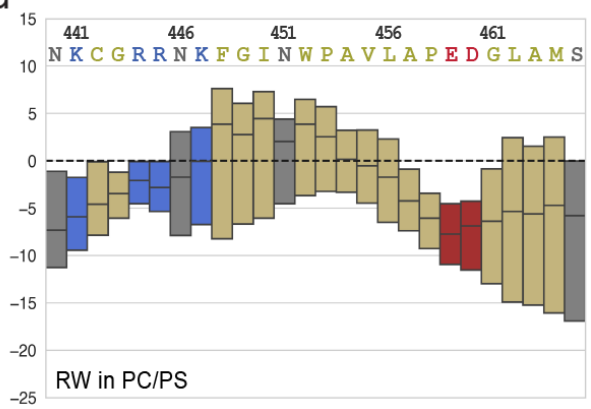

Figure S3. Insertion distance for a) WT, b) dKFG, c) FS, d) RW in PC/PS membrane. 


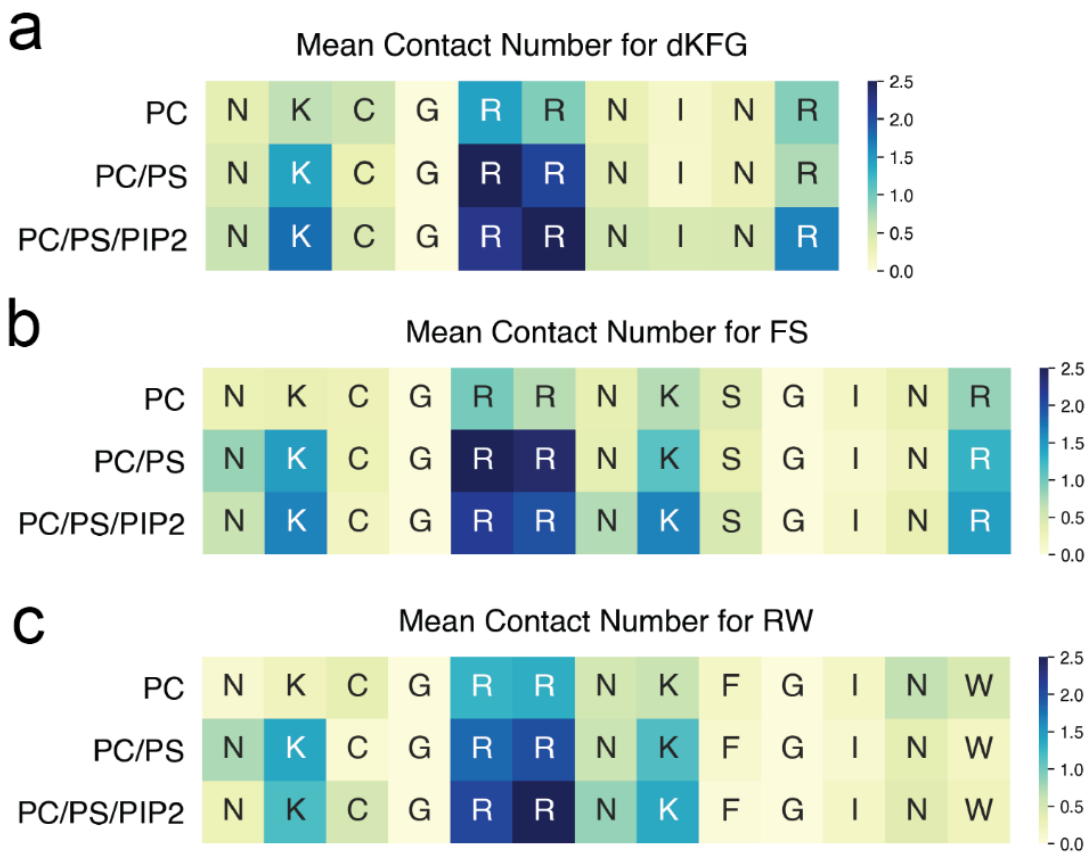

Figure S4. Mean contact number for a) dKFG, b) FS, c) RW.

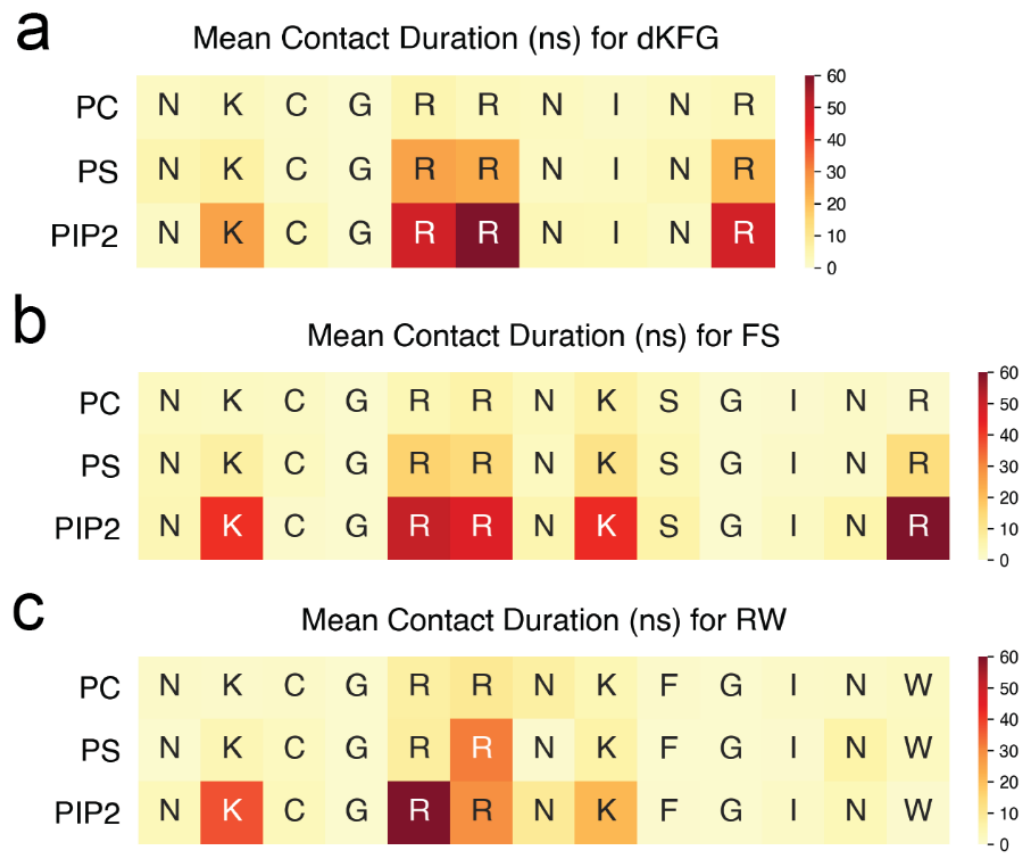

Figure S5. Mean contact duration for a) dKFG, b) FS, c) RW. 


\section{S-4 Single Vesicle TIRF Imaging}

\section{Synthesize of vesicles}

Vesicles with different compositions were synthesized according to established protocols. ${ }^{17}$ Glass vials and glass syringes were washed three times with Milli-Q water, ethanol (100\%, Fisher Scientific, USA) and chloroform (Fisher Scientific, USA) before use. Lipids (Avanti Polar Lipids, Inc, USA) were added into the cleaned glass vial by the glass syringe and dried under vacuum overnight. The glass vial was wrapped with alumina foil with a small hole on the top. Upon drying, the formed film on the glass vial was dissolved with $200 \mu \mathrm{L}$ of $50 \mathrm{mM}$ tris buffered saline $(\mathrm{pH}$ 8.0). The synthesized vesicle was diluted to designated concentrations and sonicated by an ultrasonic liquid processor (Misonix, USA) for 2 min (amplitude: 10\%, process time: $2 \mathrm{~s}$, and quiet time: $4 \mathrm{~s}$ ).

Table S4. Vesicle Compositions

\begin{tabular}{|c|c|c|c|c|}
\hline & DOPC (mol\%) & DOPS (mol\%) & PiP2 (mol\%) & Biotin-PE (mol\%) \\
\hline Vesicle 1 & 99.8 & 0 & 0 & 0.2 \\
\hline Vesicle 2 & 64.8 & 35 & 0 & 0.2 \\
\hline Vesicle 3 & 94.8 & 0 & 5 & 0.2 \\
\hline
\end{tabular}

\section{Prepare of the biotin-PEG coated quartz slides and cover slides}

The quartz slides (with drilled holes, 1 inch $\times 3$ inch, $1 \mathrm{~mm}$ thick, Finkenbeiner Inc, USA) and the cover slides (24 mm $\times 40 \mathrm{~mm}$, Corning, USA) were coated with biotin-polyethylene glycol (biotin-PEG) and PEG in order to eliminate nonspecific binding of vesicles, as well as to generate biotin-NeutrAvidin bridges on the surface. The biotin-PEG and PEG were covalently immobilized onto the slides surface according to the established protocol. ${ }^{18}$ Briefly, the slides were thoroughly cleaned with household detergent, MilliQ water, acetone (Fisher Scientific, USA), $1 \mathrm{M}$ potassium hydroxide (Fisher Scientific, USA) and methanol (99.8\%, Fisher Scientific, USA) for $1 \mathrm{~h}$ each. Each quartz slide was burnt with a propone torch, incubated in methanol containing with 1\% (v/v) 3-Aminopropyltriethoxysilane (APTES, Sigma, USA) and 5\% (v/v) acetone, and coated with m-PEG-SVA and biotin-PEG-SVA (Laysan Bio Inc, USA). The flow chamber was assembled from the biotin-PEG coated quartz slide and a cover slide using double sided tape and Epoxy glue.

Immobilize of bio-samples onto the flow chamber 
Incubate $50 \mu \mathrm{L}$ of $0.1 \mathrm{mg} / \mathrm{mL}$ NeutrAvidin (Fisher Scientific, USA) in the channels for $5 \mathrm{~min}$ at room temperature, followed by a thorough washing of the unbound NeutrAvidin. Incubate $50 \mu \mathrm{L}$ of vesicles in the channel for $30 \mathrm{~min}$ at room temperature and wash out unbound vesicles with $200 \mu \mathrm{L}$ T50. Inject $50 \mu \mathrm{L}$ of Cy3 labeled RW, FS or WT peptides (78.0\%, 90.0\% and 75.3\%, Genscript, China) into the channel and incubate for $15 \mathrm{~min}$ at room temperature and thoroughly wash out unbound peptides with $200 \mu \mathrm{L}$ T50 buffer. Before imaging, $50 \mu \mathrm{L}$ of oxygen scavenger solution $(0.1 \mathrm{mg} / \mathrm{mL}$ glucose oxidase (Sigma, USA), 0.02 $\mathrm{mg} / \mathrm{mL}$ catalase (Sigma, USA) and $0.8 \%(\mathrm{w} / \mathrm{w})$ dextrose (sigma, USA), $3 \mathrm{mM}$ 6-hydroxy-2,5,7,8tetramethylchroman-2-carboxylic acid (trolox, sigma, USA)) was injected into each channel to eliminate single-molecule blinking.

\section{Single-Molecule Imaging Through Total Internal Reflection Fluorescence Microscopy (TIRF)}

The binding events between the vesicles and the peptides were recorded with TIRF microscopy. DiD labeled vesicles were excited at $633 \mathrm{~nm}$ and the Суз labeled peptides were excited at $532 \mathrm{~nm}$. Twenty frames of image stack were taken with $200 \mathrm{~ms}$ exposure time. Real-time image analysis was done using the custom software obtained from Dr. Taekjip Ha's group at the Johns Hopkins University. 


\section{S-5 TIRF Imaging Supplementary Figures}

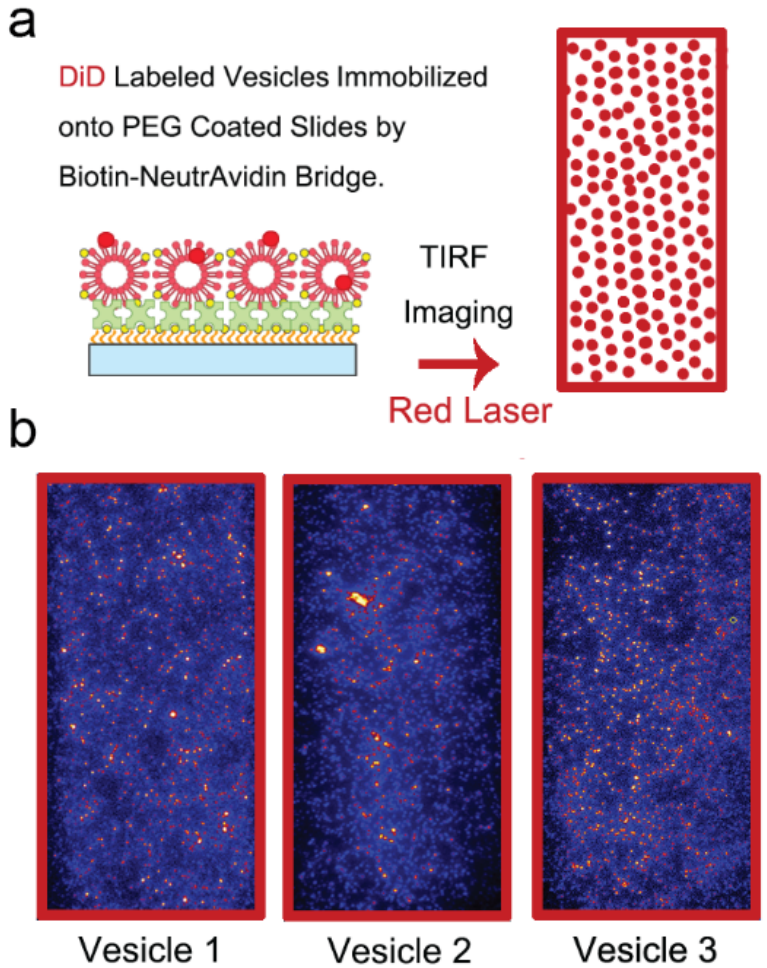

Figure S6. Vesicle immobilized onto the slides surface through a biotinNeutrAvidin bridge with a full coverage. (a) Schematic illustration of evaluation of the homogeneity and surface coverage of the vesicle layer using red laser illuminated at $633 \mathrm{~nm}$. (b) Representative results of the DiD labeled vesicle layers on the substrate imaging by TIRF Microscopy with the vesicle concentration of 100 $\mu \mathrm{M} .2 \mathrm{~mol} \%$ DiD were used to substitute DOPC for labeling vesicles listed in Table S4. 


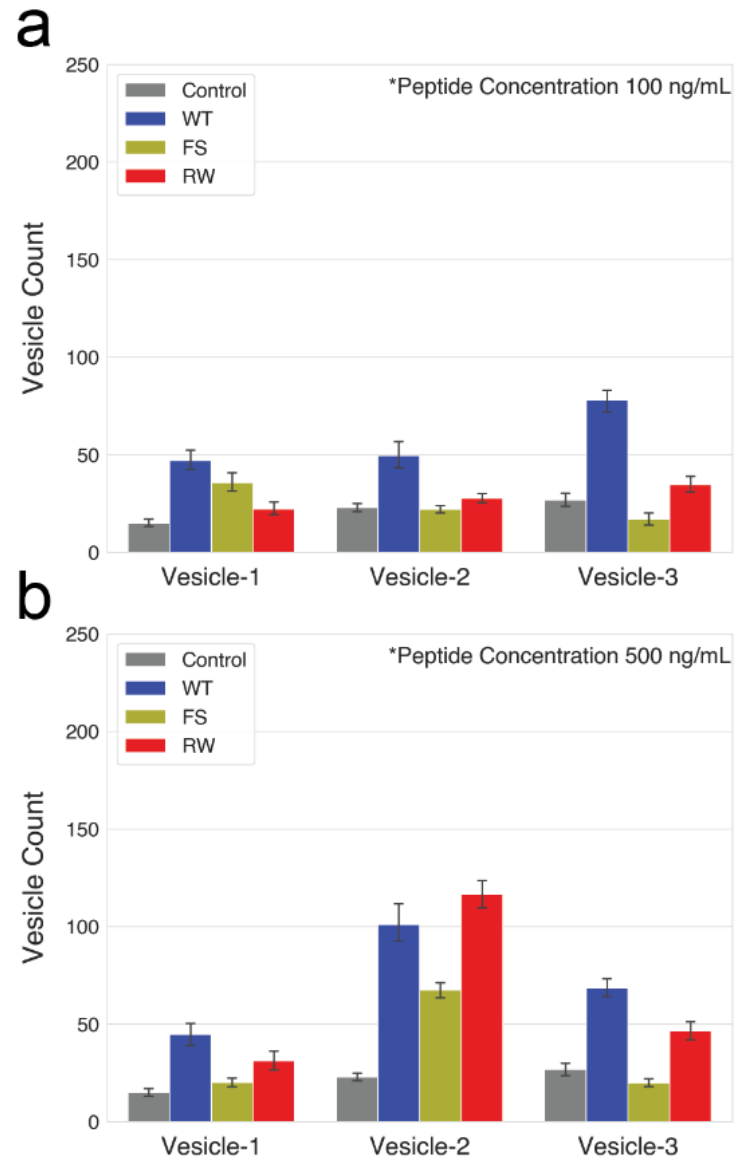

Figure S7. Fluorophore counts of Cy3-labeled peptides bound with different vesicles. (a) Vesicle count at peptide concentrations of $100 \mathrm{ng} / \mathrm{mL}$. (b) Vesicle count at peptide concentrations of $500 \mathrm{ng} / \mathrm{mL}$. At $500 \mathrm{ng} / \mathrm{mL}$, vesicle-2-PC/PS shows observable binding and the trend is consistent with the result from 1 $\mu \mathrm{g} / \mathrm{mL}$ 


\section{S-6 In-cell Experiment}

\section{Cell culture and transfection}

SH-SY5Y cells were cultured in DMEM/F12K 50/50 medium supplemented with $10 \%$ FBS, and $1 \times$ Penicillin-Streptomycin solution (complete medium) in $35 \mathrm{~mm}$ dish. Cultures were maintained in a standard humidified incubator at $37^{\circ} \mathrm{C}$ with $5 \% \mathrm{CO}$. For Western blots, $600 \mathrm{ng}$ of DNA were combined with $1.8 \mathrm{ul}$ Turbofect in $60 \mathrm{ul}$ of serum-free DMEM/F12K 50/50. The transfection mixtures were incubated at room temperature for 20 minutes prior to adding to cells cultured in $35 \mathrm{~mm}$ dishes with $2 \mathrm{~mL}$ complete medium. The transfection medium was replaced with $2 \mathrm{~mL}$ complete medium after 3 hours of transfection to recover cells overnight. As for HEK293T cells, the medium for culture was DMEM supplemented with 10\% FBS, and $1 \times$ Penicillin-Streptomycin solution (complete medium), transfection mixtures were made with serumfree DMEM.

\section{Western Blot}

SH-SY5Y Cells were washed once with $1 \mathrm{~mL}$ cold DPBS and changed to $1 \mathrm{~mL}$ serum-free DMEM/F12K 50/50 medium with 1x Penicillin-Streptomycin solution to minimize the base-level ERK activation induced by serum. After starvation for 5 hours, $20 \mu \mathrm{g}$ Cycloheximide was added directly to the medium and incubated for $1 \mathrm{~h}$ before NGF stimulation (1000 $\mathrm{ng} / \mathrm{mL}$ final concentration). Cells were then lysed with $100 \mu \mathrm{L}$ cold lysis buffer (RIPA + protease/phosphatase cocktail) at $0,10,90,180$ minutes. Lysates were centrifuged at $17,000 \mathrm{RCF}$ for $10 \mathrm{~min}$ at $4^{\circ} \mathrm{C}$ and the suspension was collected. Purified lysates were normalized using the Bradford reagent (Thermo Fisher Scientific \#23238). Equal amount of samples was mixed with LDS buffer and loaded onto $10 \%$ or $12 \%$ polyacrylamide gels. SDS-PAGE was performed at room temperature with cold water bath. Samples were transferred to PVDF membranes at 30 $\mathrm{V}$ overnight or $80 \mathrm{~V}$ for $90 \mathrm{~min}$ at $4^{\circ} \mathrm{C}$. Membranes were blocked in $5 \% \mathrm{BSA} / \mathrm{TBST}$ for $1 \mathrm{~h}$ at room temperature and probed with the primary and secondary antibodies according to manufacturer's protocol. Membranes were incubated with ECL substrate and imaged using a Bio-Rad ChemiDoc XRS chemiluminescence detector (BioRad). As for HEK293T cells, serum-free DMEM with 1× PenicillinStreptomycin solution was used for starvation, and all the rest procedure was the same.

\section{Quantification of protein level}

The band intensity was analyzed by ImageJ. The level of HA-TrkA and pERK was normalized with intensity of GAPDH in each lane. Student t-test was done using Graphpad Prism. 


\section{S-7 In-cell Experiment Supplementary Figures}

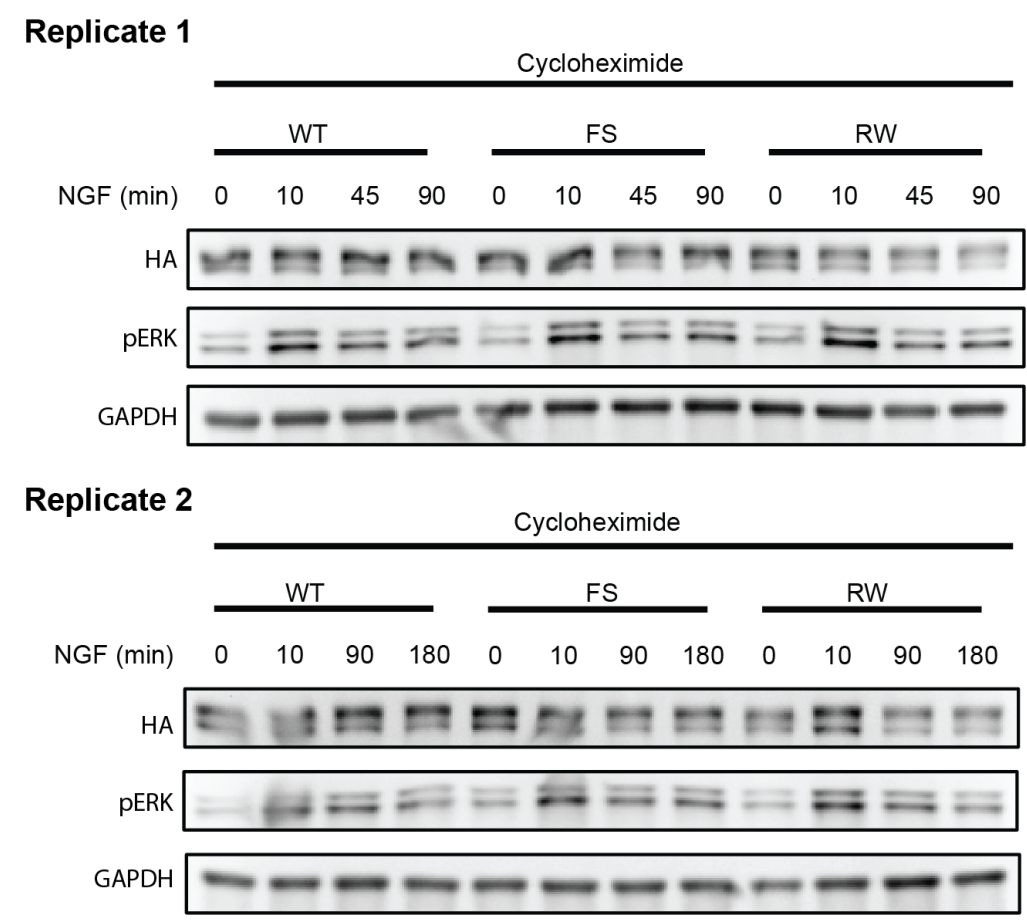

Figure S8. Two additional replicates for Western blot analysis of TrkA abundance and ERK activity from SH-S5Y5, used to generate Figure $5 \mathrm{~b}, \mathrm{~d}$.

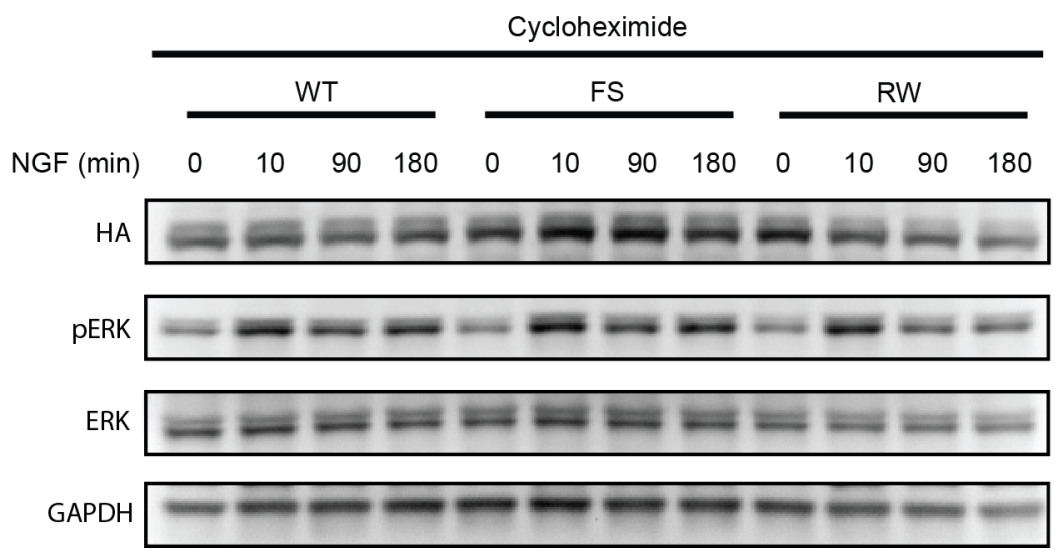

Figure S9. Quantification of HA and pERK level for WT, FS, and RW HA-hTrkA transfected in HEK293 cell-line. Total ERK and loading control GADPH levels are mostly invariant. Receptor signaling activity probed by pERK level does not show observable difference. Receptor degradation is not evident in this cell-line, suggesting the lack of degradation machinery. 


\section{REFERENCES}

(1) The Uniprot Consortium. UniProt: A Worldwide Hub of Protein Knowledge. Nucleic Acids Res. 2019, 47, 506-515. https://doi.org/10.1093/nar/gky1049.

(2) Hanwell, M. D.; Curtis, D. E.; Lonie, D. C.; Vandermeerschd, T.; Zurek, E.; Hutchison, G. R. Avogadro: An Advanced Semantic Chemical Editor, Visualization, and Analysis Platform. J. Cheminform. 2012, 4, 17. https://doi.org/10.1186/1758-2946-4-17.

(3) Jo, S.; Kim, T.; Iyer, V. G.; Im, W. CHARMM-GUI: A Web-Based Graphical User Interface for CHARMM. J. Comput. Chem. 2008, 29, 1859-1865. https://doi.org/10.1002/jcc.20945.

(4) Ohkubo, Y. Z.; Pogorelov, T. V.; Arcario, M. J.; Christensen, G. A.; Tajkhorshid, E. Accelerating Membrane Insertion of Peripheral Proteins with a Novel Membrane Mimetic Model. Biophys. J. 2012, 102 (9), 2130-2139. https://doi.org/10.1016/j.bpj.2012.03.015.

(5) Pogorelov, T. V.; Vermaas, J. V.; Arcario, M. J.; Tajkhorshid, E. Partitioning of Amino Acids into a Model Membrane: Capturing the Interface. J. Phys. Chem. B 2014, 118 (6), 1481-1492. https://doi.org/10.1021/jp4089113.

(6) Vermaas, J. V.; Baylon, J. L.; Arcario, M. J.; Muller, M. P.; Wu, Z.; Pogorelov, T. V.; Tajkhorshid, E. Efficient Exploration of Membrane-Associated Phenomena at Atomic Resolution. J. Membr. Biol. 2015, 248 (3), 563-582. https://doi.org/10.1007/s00232-015-9806-9.

(7) Qi, Y.; Cheng, X.; Lee, J.; Vermaas, J. V.; Pogorelov, T. V.; Tajkhorshid, E.; Park, S.; Klauda, J. B.; Im, W. CHARMM-GUI HMMM Builder for Membrane Simulations with the Highly Mobile Membrane-Mimetic Model. Biophys. J. 2015, 109 (10), 2012-2022.

https://doi.org/10.1016/j.bpj.2015.10.008.

(8) Phillips, J. C.; Braun, R.; Wang, W.; Gumbart, J.; Tajkhorshid, E.; Villa, E.; Chipot, C.; Skeel, R. D.; Kalé, L.; Schulten, K. Scalable Molecular Dynamics with NAMD. J. Comput. Chem. 2005, 26 (16), 1781-1802. https://doi.org/10.1002/jcc.20289.

(9) Huang, J.; Rauscher, S.; Nawrocki, G.; Ran, T.; Feig, M.; De Groot, B. L.; Grubmüller, H.; MacKerell, A. D. CHARMM36m: An Improved Force Field for Folded and Intrinsically Disordered Proteins. Nat. Methods 2016, 14, 71-73. https://doi.org/10.1038/nmeth.4067.

(10) Jorgensen, W. L.; Chandrasekhar, J.; Madura, J. D.; Impey, R. W.; Klein, M. L. Comparison of Simple Potential Functions for Simulating Liquid Water. J. Chem. Phys. 1983, 79 (2), 926. https://doi.org/10.1063/1.445869. 
(11) K. Vanommeslaeghev, E. Hatcher, C. Acharya, S. Kundu, S. Zhong, J. Shim, E. Darian, O. Guvench, P. Lopes, I. Vorobyov, Mackerell Jr., A. D. CHARMM General Force Field: A Force Field for Drug-Like Molecules Compatible with the CHARMM All-Atom Additive Biological Force Fields. J. Comput. Chem. 2010, 31 (16), 2967-2970. https://doi.org/10.1002/jcc.

(12) Essmann, U.; Perera, L.; Berkowitz, M. L.; Darden, T.; Lee, H.; Pedersen, L. G. A Smooth Particle Mesh Ewald Method. J. Chem. Phys. 1995, 103, 8577. https://doi.org/10.1063/1.470117.

(13) Miyamoto, S.; Kollman, P. A. Settle: An Analytical Version of the SHAKE and RATTLE Algorithm for Rigid Water Models. J. Comput. Chem. 1992, 13 (8), 952-962.

https://doi.org/10.1002/jcc.540130805.

(14) Martyna, G. J.; Tobias, D. J.; Klein, M. L. Constant Pressure Molecular Dynamics Algorithms. J. Chem. Phys. 1994, 101 (5), 4177-4189. https://doi.org/10.1063/1.467468.

(15) Humphrey, W.; Dalke, A.; Schulten, K. VMD: Visual Molecular Dynamics. J. Mol. Graph. 1996, 14 (1), 33-38. https://doi.org/10.1016/0263-7855(96)00018-5.

(16) Michaud-Agrawal, N.; Denning, E. J.; Woolf, T. B.; Beckstein, O. MDAnalysis: A Toolkit for the Analysis of Molecular Dynamics Simulations. J. Comput. Chem. 2011, 32 (10), 2319-2327. https://doi.org/10.1002/jcc.21787.

(17) Cai, B.; Yu, L.; Sharum, S. R.; Zhang, K.; Diao, J. Single-Vesicle Measurement of Protein-Induced Membrane Tethering. Colloids Surfaces B Biointerfaces 2019, 177 (November 2018), 267-273. https://doi.org/10.1016/j.colsurfb.2019.02.004.

(18) Diao, J.; Ishitsuka, Y.; Lee, H.; Joo, C.; Su, Z.; Syed, S.; Shin, Y. K.; Yoon, T. Y.; Ha, T. A Single Vesicle-Vesicle Fusion Assay for in Vitro Studies of Snares and Accessory Proteins. Nat. Protoc. 2012, 7 (5), 921-934. https://doi.org/10.1038/nprot.2012.020. 